\title{
ANALYSIS OF PROPERTIES OF A LUNG MECHANICAL MODEL DURING ARTIFICIAL VENTILATION USING MEASUREMENT STATION
}

\author{
Kamil Jakuszkin \\ Wroclaw University of Technology, Department of Electronics, B. Prusa 53/55, 50-317 Wroclaw, Poland( $₫$ kamil.jakuszkin@ pwr.wroc.pl, \\ +4871320 6329)
}

\begin{abstract}
The paper shows the usefulness of the lung mechanical model for time and frequency characteristics reconstruction proper for the mechanics of an adult human respiratory system in its various regimes of work. The complex set-up for measurements of human respiratory system mechanics is presented. Two separate scenarios were created, firstly, the mechanical model was examined using standard mechanical ventilation routine with embedded Interrupter Technique and then the Optimized Ventilator Waveform technique was tested. An analysis of experimental results is presented, as well as an outline of the issues and problems revealed during investigations.
\end{abstract}

Keywords: lungs mechanical model, Interrupter Technique, Optimized Ventilator Waveform, artificial ventilation.

(C) 2010 Polish Academy of Sciences. All rights reserved

\section{Introduction}

The human respiratory system is one of the most important elements in maintaining an organism's vital functions. The large complexity of its mechanics is a well-known issue [1, 2]. A regular and healthy function of all the elements depends on many mechanical and physiological factors, however there are pathologies which disturb the symmetry in this natural organisation.

There is a need to identify and specify system parameters properly in order to recognize and learn the occurring phenomena [3]. System complexity implicates the analysis of changes on many levels and compartments. The choice of correct treatment or therapy depends on that identification. Generally, measurement techniques can be divided into two groups: those which require active patient participation (medical diagnostics, spirometry, etc.) and those used during mechanical (supported) ventilation. In both cases the main aim is to minimize the pain and active participation of the patient. Standard procedures are based on pressure and airflow measurements in proximal part of the respiratory system (mouth). Those pressure/flow measurements are conducted in many scenarios and configurations in order to extract different types of data (i.e. time, frequency domain [4, 5]). During mechanical ventilation, the acquired signals are used in control feedback and also in real time monitoring $[6,7]$.

One of the ways of research development within respiratory mechanics studies is the experiment on a real object. In medical sciences the real object experiment is as important as mathematical model simulations [8]. Better understanding of the human respiratory system mechanics have enabled the creation of the artificial lung (mechanical model) which is capable of imitating real object behaviour. The aim of the paper was to assess the usefulness of a chosen mechanical model for testing the available experimental measurement methods. 
Two techniques were used for verification [9] of Dual Adult PNEUVIEW 5600i mechanical model manufactured by Michigan Instruments. The first of them, the Interrupter Technique [10] was embedded in a mechanical ventilation cycle. The second one, Optimized Ventilator Waveform $(\mathrm{OVW})$, was treated as a generic ventilator mode $[6,11]$.

\section{Methods and algorithms}

\subsection{Optimized Ventilator Waveform}

In order to conduct tests on artificial lungs, the Optimized Ventilator Waveform (OVW) technique was used. It allows to determine system impedance in the frequency domain. The OVW derives from Forced Oscillation Technique (FOT) [12] and incorporates some improvements, like time saving or therapy and measurement task optimization. Both techniques calculate the input impedance $Z_{r s}$ using pressure and flow signals recorded in the proximal part of the respiratory system. The OVW is both part of artificial ventilation routine as well as the measurement tool and assumes generation of a specific flow pattern given by Eq. (1):

$$
Q=\sum_{i=1}^{7} A_{i} \cos \left(2 \pi f_{i} t+\varphi_{i}\right) .
$$

Flow pattern $Q$ is the sum of seven cosine functions which meet the "non sum non difference" (NSND) condition [6]. The exact function parameters are shown in Table 1.

Table 1. The OVW flow pattern parameters.

\begin{tabular}{|c|c|c|c|}
\hline$i$ & Amplitude $A$ & Frequency $f$ & Phase $\varphi$ \\
\hline 1 & 1 & 0.156250 & 4.95 \\
\hline 2 & 0.3 & 0.390625 & 3.82 \\
\hline 3 & 0.25 & 0.859375 & 4.37 \\
\hline 4 & 0.25 & 1.484375 & 3.67 \\
\hline 5 & 0.175 & 2.421875 & 4.05 \\
\hline 6 & 0.175 & 4.609375 & 4.13 \\
\hline 7 & 0.125 & 8.046875 & 4.02 \\
\hline
\end{tabular}

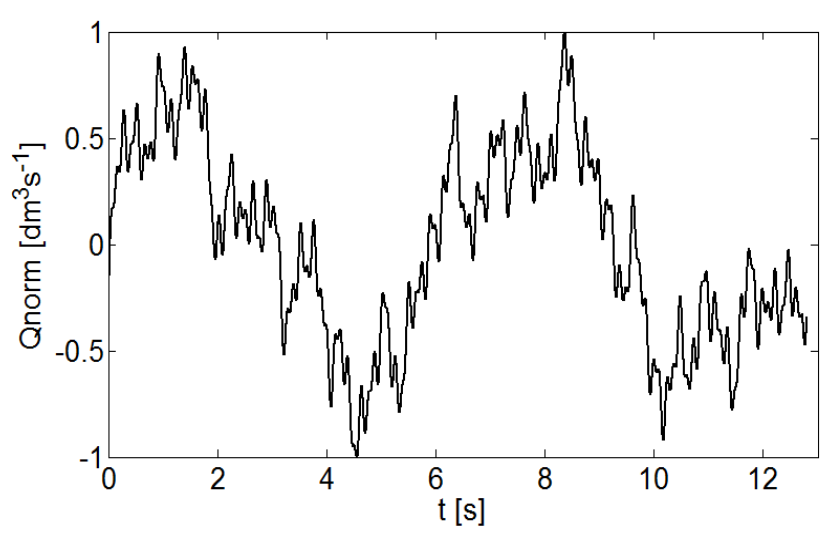

Fig. 1. Normalized OVW flow pattern.

The above parameters guarantee a spectrum leak reduction and overall volume at a level of $0.5 \mathrm{dm}^{3}$. The generated flow pattern incorporates two full breaths in a time of $12.8 \mathrm{~s}$. All these conditions were applied during the experiment organized in the repeated research and the 
example source signal was presented in Fig. 1. The flow pattern was normalized in order to acquire eligible volume levels.

Generally in ventilation supporting respiratory resistances are formulated as a proportion between pressure change $\Delta P$ (effect) to driven flow value $Q$ (cause) and are given by Eq. (2):

$$
R=\frac{\Delta P}{Q}
$$

In literature, there are prepared algorithms devoted to the calculation of coupled respiratory system impedance on the basis of time domain data of flow and pressure [13]. In the presented work, sampled values of acquired signals are the system responses to the OVW source pattern. Fixing the number of samples on $2 \mathrm{n}$ for the whole measurement period (12.8 s) enabled reliable calculation for pressure $\left(P_{F F T}(f)\right)$ and flow $\left(Q_{F F T}(f)\right)$ trends [6]. In the designed experiment the sampling frequency $f_{s}$ was set to $640 \mathrm{~Hz}$. The corresponding power spectrum density of the pressure signal $G_{P P}(f)$ and the cross power spectrum of pressure and flow data $G_{Q P}(f)$ can be then calculated according to formulas (3) and (4) respectively:

$$
\begin{aligned}
& G_{P P}(f)=P_{F F T}(f) \cdot P_{F F T}(f) *, \\
& G_{Q P}(f)=Q_{F F T}(f) \cdot P_{F F T}(f) *,
\end{aligned}
$$

where $P_{F F T}(f)^{*}$ stands for the conjugate value of $P_{F F T}(f)$.

Based on the results, the complex respiratory impedance $Z_{r s}(f)$ was calculated:

$$
Z_{r s}(f)=\frac{G_{P P}(f)}{G_{Q P}(f)} .
$$

The whole signal energy is focused now on seven harmonics of the OVW waveform so that the real and imaginary part of $Z_{r s}$ is calculated for frequencies listed in Table 1:

$$
\begin{gathered}
\operatorname{Re}\left(Z_{r s}(f)\right)=R(f), \\
\operatorname{Im}\left(Z_{r s}(f)\right)=E(f)=C^{-1}(f) .
\end{gathered}
$$

The real part (6) represents the resistive $(R(f))$ character of the examined system, whereas the imaginary component (7) reflects its elastic $(E(f))$ behavior (which can be also interpreted in sense of compliance $C=E^{-1}$ ).

\subsection{Interrupter Technique}

Proposed by von Neergard and Wirtz, the airflow Interrupter Technique (IT) uses proximal pressure and flow signals which are recorded during the occlusion [14]. The IT is successfully used during respiratory system mechanics diagnostics [15]. Main advantages of the IT algorithm are its small invasiveness, minimal requirements regarding patient cooperation and also low implementation cost. The one diagnostically useful parameter in the IT technique is the interrupter resistance $R_{\text {int }}$. However, research on expanding IT utility (promising frequency domain analysis results) is being conducted [4]. The main problem with the IT is a low accuracy and repeatability of $R_{\text {int }}$ evaluations.

Harmless embedding of the airflow Interrupter Technique into mechanical ventilation routine seems to be possible and justified [7]. Especially interesting can be a fusion of OVW advantages as a ventilation scheme and enhanced IT diagnostic procedure [3]. For this reason, 
the occlusion moment was placed at the end of the inhalation (synchronized with the piston movement) of controlled ventilation scenario in the designed experiment.

The standard version of the IT measurement protocol generates quick flow interruption (100 ms standard) and results in fast airway opening pressure rise $P_{a o}$ [16]. An exemplary plot is shown in Fig. 2.

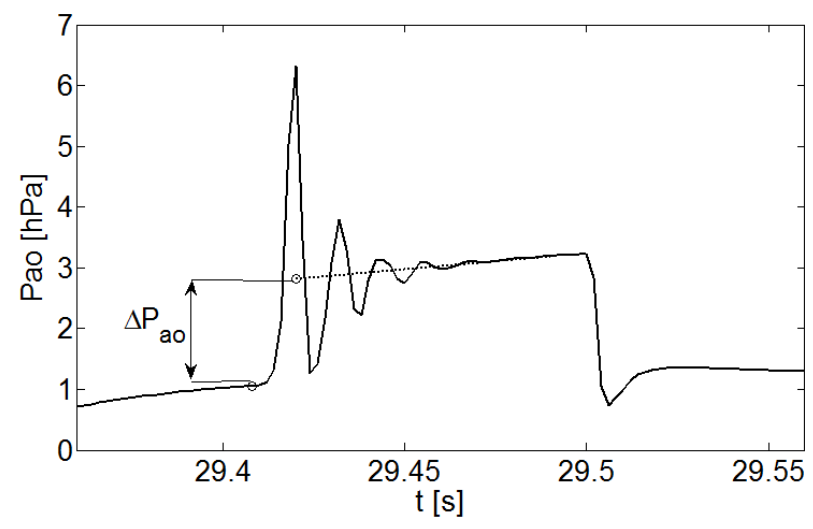

Fig. 2. The flow waveform during the moment of occlusion.

Estimation of parameter $R_{\text {int }}$ during artificial ventilation can be considered as a single task of inverse problem solution $[17,15]$.

The algorithm is based on the assumption that respiratory system mechanics is not fast enough to react to rapid flow interruption and the airway opening pressure $P_{a o}$ during occlusion automatically rises to the alveolar level $\left(P_{A}\right)$ [10]. In conclusion, knowing the initial pressure step $\Delta P_{A}$ and the pre-occlusion $Q$ value, $R_{\text {int }}$ can be calculated according to (2).

Some of the accompanying processes (i.e. the time needed for pressure equilibration, tissue relaxation) are neglected which reflects in overestimation of $R_{\text {int }}$ (about $20 \%$ ).

Additional difficulties occur with the initial rapid rise of $P_{a o}$ value. For that reason the exact value of $\Delta P_{a o}$ is estimated as the result of back extrapolation of the second rise phase on the first one (Fig. 2 dotted line). The back extrapolation was accomplished by selecting two characteristic points (and their neighbourhoods) of the second phase. Sampling was made around the 30-th and 70-th ms of occlusion duration. The algorithm estimated the $P_{a o}\left(t_{c}\right)$ in 20-th ms of occlusion as the moment $\left(t_{c}\right)$ of vale full closure. The $P_{a o}$ initial rise moment $\left(t_{i}\right)$ was selected basing on the "start of occlusion" control sample data. The final $R_{\text {int }}$ value is given by equation:

$$
R_{\mathrm{int}}=\frac{P_{a o}\left(t_{c}\right)-P_{a o}\left(t_{i}\right)}{Q\left(t_{i}\right)} .
$$

\subsection{Hardware}

The Training Test Lung TTL manufactured by Michigan Instruments (Fig. 3) is part of the complex station for measurements of human respiratory system parameters. In the presented research the following modules were used [18]:

- Jaeger flow and pressure transducers for medical use;

- Keithley KPCI3108 data acquisition card;

- low frequency ventilator ( $1.1 \mathrm{dm}^{3}$ maximum working volume);

- PC class computer with LabView 6.1. software. 
The TTL mechanical model consists of two independent bellows of overall volume $2 \mathrm{dm}^{3}$ and additional $1.022 \mathrm{dm}^{3}$ dead space.

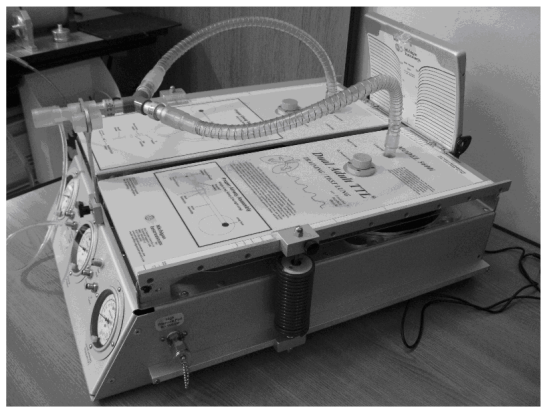

Fig. 3. The TTL unit used during research.

The adjustable elements allow the simulation of various scenarios. There are three regulation-dependent sections: upper airways resistance, bronchi resistance and lung elastance (see Fig. 4 and Fig. 5).

Resistance can be adjusted by replacing flow limiting resistors. The resistors provided with TTL are flow dependent in a non-linear way (parabolic) and the appropriate values were evaluated using technical documentation and reference measurements.

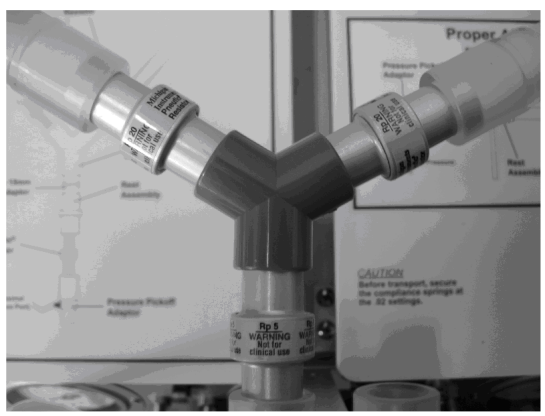

Fig. 4. Adjustable resistive elements modeling the upper airways and bronchial resistance.

Lungs compliance can be adjusted using two springs (one for each bellow). In the beginning of the breathing maneuver, springs generate a rapid increase of bellow pressure which can generate noise in further signal analysis. For that reason every maneuver begins with pre-stretched springs.

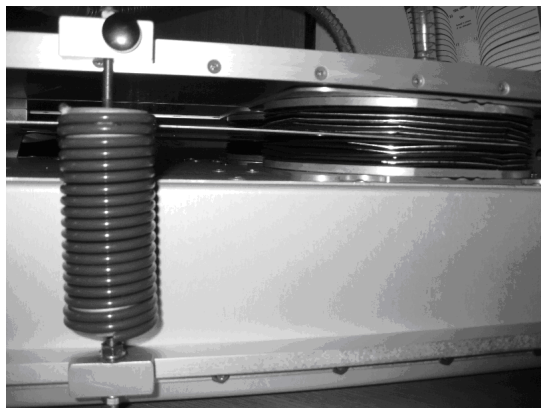

Fig. 5. Adjustable elastic spring modeling lung compliance. 
The important part of the IT measurement module is a medical purpose pressure sensor with embedded interrupter valve (Fig. 6). An electromagnetic shutter assures a closure time of $\sim 20 \mathrm{~ms}$ which is sufficient to conduct the routine properly.

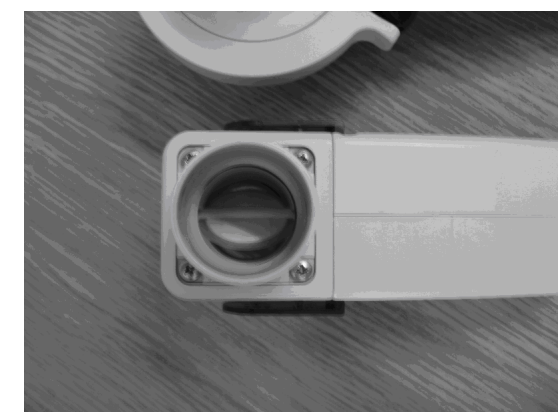

Fig. 6. Medical purpose pressure sensor with embedded shutter.

The main advantage of the solution is the valve's ability to sustain tightness during closure by continuous actuation of the shutter [18]. For IT experiment purposes, control signals responsible for the piston and shutter operation were generated at a rate of $1 \mathrm{kHz}$. The main aim of the author was to maintain full synchronization between ventilator piston movement and moments of flow interruption occurrences. A detailed synchronization moment is shown in Fig. 7.

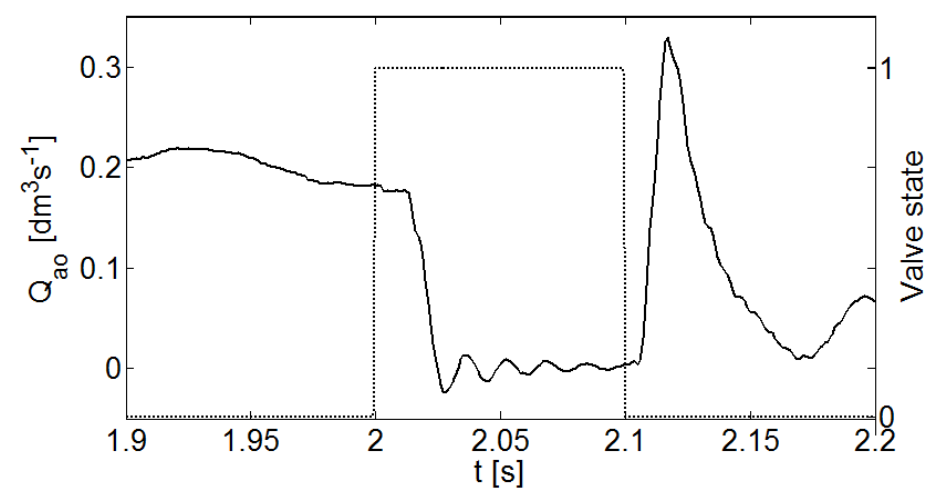

Fig. 7. A moment of ventilator-valve synchronization: solid line - flow $Q_{a o}$; dotted line - valve state.

\section{Results}

The main aim of the author was to assess the working conditions of lungs mechanical model and analyze its behavior during different ventilatory routines. The series of tests were conducted and the results are presented. The described mechanical model was tested during two ventilation scenarios. In the first one, lungs were ventilated using OVW pattern (under various parameter values). In the second one, the standard controlled ventilation cycle with the additional flow interruptions was commenced.

Some test procedures were conducted in order to obtain results on parabolic resistor behavior in simulated scenarios after software and hardware calibration. For constant flow value a series of five measurements for each resistor were done. The results are presented in Table 2. 
Table 2. Parabolic resistor values for constant flow value.

\begin{tabular}{|c|c|c|}
\hline Resistor symbol & $\begin{array}{c}\text { Mean Resistance } \\
{\left[\mathrm{hPa} / \mathrm{dm}^{3} / \mathrm{s}\right]}\end{array}$ & $\begin{array}{c}\text { Error } \\
{[\%]}\end{array}$ \\
\hline $\mathrm{Rp} 20$ & 3.8 & 0.4 \\
\hline $\mathrm{Rp} 50$ & 20.8 & 0.1 \\
\hline
\end{tabular}

For the above results the flow value was always $0.2 \mathrm{dm}^{3} / \mathrm{s}$ (which was a further IT experiment condition). The error was calculated as a doubled value of mean resistance standard deviation $(S D)$. The tested resistors were used in a controlled ventilation experiment.

The OVW experiment required research on resistor behavior under dynamically changing flow pattern. The resistors were tested individually using the Optimized Waveform shown in Fig. 1. In order to simplify the modeling process, mean resistances for the whole frequency range were used. Recorded resistances were averaged by frequency $f$ (Table 1) in each of three measurement series. The three series average value was used in the model. The results are shown in Table 3.

Table 3. Parabolic resistors values for dynamic flow pattern.

\begin{tabular}{|c|c|c|c|}
\hline Resistor symbol & $\begin{array}{c}\text { Mean Resistance } \\
{\left[\mathrm{hPa} / \mathrm{dm}^{3} / \mathrm{s}\right]}\end{array}$ & $\begin{array}{c}\Delta R \\
{\left[\mathrm{hPa} / \mathrm{dm}^{3} / \mathrm{s}\right]}\end{array}$ & $\begin{array}{c}\delta R \\
{[\%]}\end{array}$ \\
\hline $\mathrm{Rp} 5$ & 1.3 & 0.4 & 33 \\
\hline $\mathrm{Rp} 20$ & 5.6 & 0.5 & 8 \\
\hline $\mathrm{Rp} 50$ & 30.3 & 3.8 & 12 \\
\hline
\end{tabular}

The error $\Delta R$ was calculated as a doubled value of series' $S D$. Tested resistors were used in OWV experiment. There is a significant difference between values for each scenario which is caused by measurement conditions changes. In few cases the flow rate was insufficient to generate the required pressure drop. The author took it under consideration during further calculations and test scenarios assembly. The Rp5 resistor which generated the most scattered results was used permanently as the proximal airways resistance model (Table 3 ) and had no significant influence on overall system resistance.

Simple electrical analog (Fig. 8) of lung model was created [19] as a reference for experimental results. Resistor $R_{p}$ can be interpreted as upper airways proximal resistance. Serial connections $R_{1}, C_{1}$ and $R_{2}, C_{2}$ stand for left and right compartment parameters.

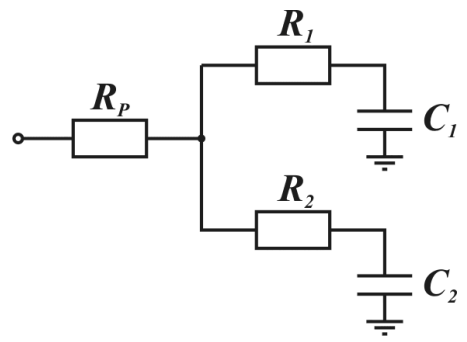

Fig. 8. Electrical model of artificial lungs verified in prepared experiment.

For the OWV scenario the author used three different presets of elements in order to simulate healthy lungs, asthma condition and emphysema. Positive pressure inside compartments was maintained during the experiment. The necessity of that maneuver aroused from the nature of metal springs modeling compliance. In the beginning of the experiment relaxed springs generated a rapid rise of pressure values and caused result inaccuracies. The 
problem was solved by keeping an initial positive pressure (and stretched springs). Fig. 9 shows correct pressure recorded with corresponding OVW flow pattern.

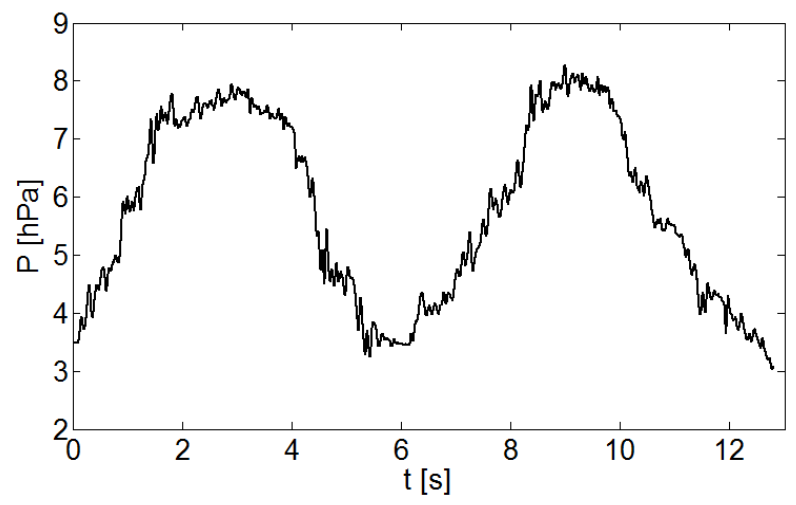

Fig. 9. Recorded pressure signal after initial spring stretch.

Five series of measurements for each condition were recorded. The OVW technique is capable of showing a pathological condition of the human respiratory system by observation of spectral characteristics changes. The condition parameters are listed in Table 4.

Table 4. Parameter values for tested conditions.

\begin{tabular}{|c|c|c|c|c|c|}
\hline Condition & $R_{p}$ & $R_{l}$ & $R_{2}$ & $\begin{array}{c}C_{I} \\
{\left[\mathrm{dm}^{3} / \mathrm{hPa}\right]}\end{array}$ & $\begin{array}{c}C_{2} \\
{\left[\mathrm{dm}^{3} / \mathrm{hPa}\right]}\end{array}$ \\
\hline Healthy & $\mathrm{Rp} 5$ & $\mathrm{Rp} 20$ & $\mathrm{Rp} 20$ & 0,05 & 0,05 \\
\hline Emphysema & $\mathrm{Rp5}$ & $\mathrm{Rp} 20$ & $\mathrm{Rp} 20$ & 0,02 & 0,02 \\
\hline Asthma & $\mathrm{Rp5}$ & $\mathrm{Rp50}$ & $\mathrm{Rp} 50$ & 0,05 & 0,05 \\
\hline
\end{tabular}

Pressure and flow signals were acquired in the proximal part of the mechanical model (which corresponds to measurements of a patient at the mouth). Healthy condition was a reference. The experiment incorporated a full OVW cycle (Fig. 1), each containing two breaths of $0.5 \mathrm{dm}^{3}$ air volume. Results are shown in Figs 10a, 10b, 11a, 11b.

During emphysema there is a significant compliance drop visible in the imaginary part of the spectrum (Fig. 10b). In that scenario the tension of the springs is greater, however resistor values are maintained on "healthy level" (Fig. 10a). Simulation results calculated using "Emphysema condition" follow the experiment results. The results assessment was based on observations of direction of the changes. From the medical point of view this significant compliance drop can be interpreted as a pathological change in tissue elasticity characteristics for diseases such as emphysema or COPD.

The "Asthma condition" was simulated in the same way. By increasing both compartment resistances the real part of the spectrum rose (Fig 11a). Elevated resistance levels also affected simulation results which follow the experiment data. Fig. 11b shows that the imaginary part of the spectrum was unaffected. The elevated resistance in the whole spectrum can indicate the bronchi shrinkage which is characteristic during an asthma attack.

The error bars ( $S D$ in Fig. 10 and Fig. 11) show that the resistive part of results is the most inaccurate element. The main cause of this state is non-linearity, which is an inherent property of elements used in experiment for imitation of different states in a real system. 
a)



b)

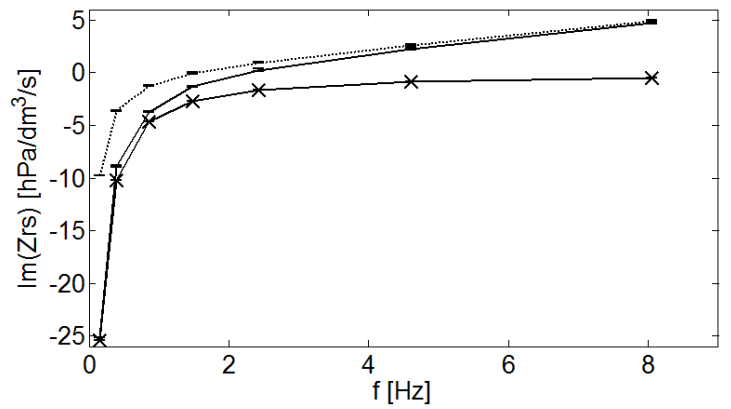

Fig. 10. Real a) and imaginary b) parts of the impedance spectrum during "Emphysema condition": x marker simulation results, solid line - "Emphysema condition", dotted line - "Healthy condition".

a)

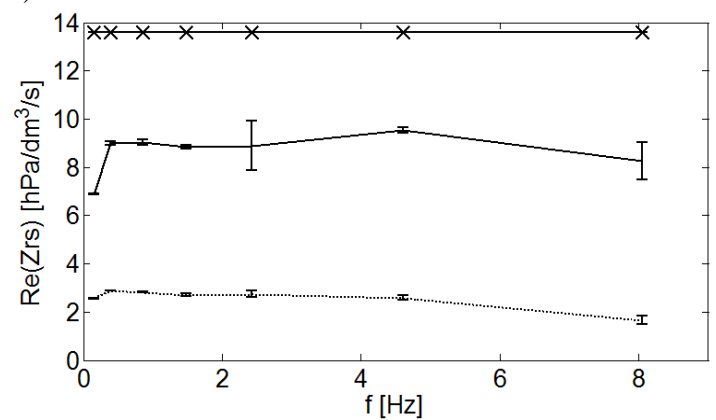

b)

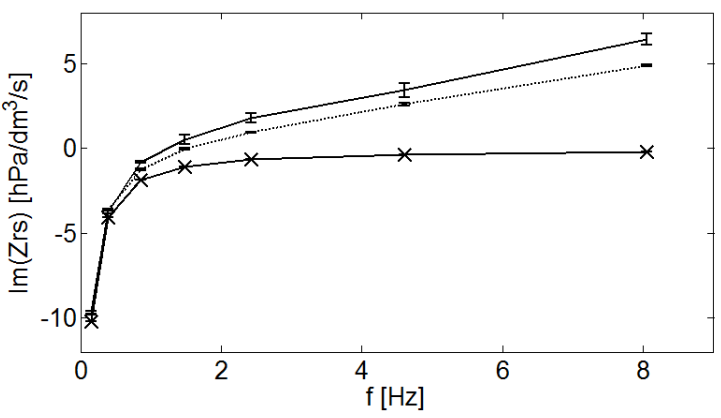

Fig. 11. Real a) and imaginary b) parts of the impedance spectrum during "Asthma condition": x marker simulation results, solid line - "Asthma condition", dotted line - "Healthy condition". 
In the IT scenario only two resistors described in Table 2 were used. Resistance $R_{p}$ with resistor Rp20 and compartment resistances $R_{1}$ and $R_{2}$ with resistor Rp50. According to chosen simulation model (Fig. 8) the total lung resistance was calculated. With the assumption that the trial was commenced in static conditions (constant flow of $0.2 \mathrm{dm}^{3} / \mathrm{s}$ ), compliances could be neglected. Therefore the reference resistance value is $14.19 \mathrm{hPa} / \mathrm{dm}^{3} / \mathrm{s}$.

Three series of twelve single breaths were conducted. Each measurement cycle contained one breath in controlled ventilation routine with $0.5 \mathrm{~s}$ pause at the end of inspiration. Single breath volume was $0.4 \mathrm{dm}^{3}$. The flow interruption was synchronized with the piston halt after inspiration.

According to the described algorithm [15], interrupter resistances $R_{\text {int }}$ for each breath were calculated. Fig. 12 shows a detailed occlusion moment with characteristic points.

The results were averaged for comparison with the model value $\left(14.19 \mathrm{hPa} / \mathrm{dm}^{3} / \mathrm{s}\right)$. Error was calculated as doubled $S D$. Deviation from the model value shows the overestimation of gathered resistances (Table 5).

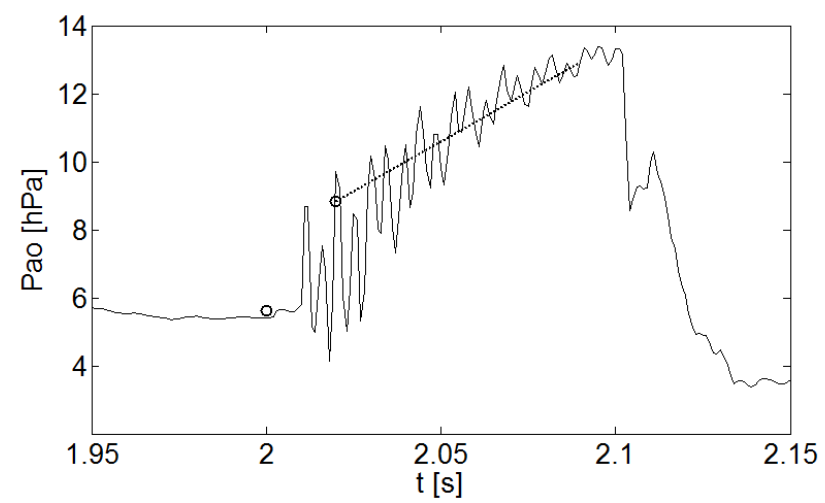

Fig. 12. The detailed occlusion moment: dotted line - back extrapolation, circles - characteristic points.

Table 5. Interrupter resistance results.

\begin{tabular}{|c|c|c|}
\hline Mean $\mathrm{R}_{\text {int }}$ & Error & Deviation form model value \\
\hline$\left[\mathrm{hPa} / \mathrm{dm}^{3} / \mathrm{s}\right]$ & {$[\%]$} & {$[\%]$} \\
\hline 15.23 & 11.91 & +7.33 \\
\hline 14.77 & 8.52 & +4.11 \\
\hline 15.40 & 7.72 & +8.55 \\
\hline
\end{tabular}

It was expected that results can be overestimated which occurs in IT measurements. The error value shows that results are repeatable over a series.

During the experiment, the author conducted another series of measurements which showed difficulties under continuous working conditions. Twelve series consisting of four complete breaths were performed. The results have shown that in every second breath there was strong overestimation $(+58.51 \%)$ of the $R_{\text {int }}$ model value. That problem could be caused by piston inertia problems and ventilator mechanical flaws. Despite this, the overall repeatability for IT measurement during artificial ventilation of mechanical model was maintained at previous level (error range 5-15\%).

\section{Conclusions}

The author of this paper described a mechanical lung model and its possible usefulness for experiential data of simulations. There are several issues that should be considered in order to 
improve the system operation. The main problem visible during a dynamical test was the parabolic nonlinearity of resistive elements. As the model was mechanically ventilated, flow values seldom exceed $0.5 \mathrm{dm}^{3} / \mathrm{s}$. Used resistors are unable to generate the required pressure drop properly in such conditions. It was the basis during the realization of the IT scenario, where the author was forced to use greater resistors to acquire the intended parameters. In future applications linear analogs will be tested. Another issue was synchronization of control signals. In a continuous experiment there were moments when the system responded incorrectly to controls. During IT routine realization made every second occlusion slightly biased which caused major overestimation of $R_{\text {int }}$. The above issue was caused by inaccurate work of pistons, that can be explained by mechanical flaws. The complex diagnostic set-up is still being developed and any construction issues are being resolved. Despite the problems author showed that during mechanical ventilation, artificial flow interruption can be easily incorporated. In future, experiments with occlusions being part of the ventilatory cycle will be conducted. The repeatability of results with the use of the mechanical model is satisfactory, however it can be improved by incorporating changes listed above.

In the dynamical OVW scenario some issues should be taken under consideration. Simulation model imperfection caused some differences that can be explained by neglecting OVW pattern dynamics and its influence on parabolic resistors operation. Hence in the "Asthma condition" the experimental real spectrum was lower than the simulated one. Spring activity is properly modeled, however simulation did not consider the inertance that affected the experimental imaginary spectrum (zero crossing and positive values). Nevertheless, the author was able to successfully commence various scenarios and show reactions for the system parameters changes. The system will be developed and new techniques and ventilatory routines will be incorporated in future work.

\section{References}

[1] I. Jabłoński, J. Mroczka: "The problem of measurement data complexity for example of the general model of the central respiratory generator and recurrent plots analysis". Metrol. Meas. Syst., vol. XV, no. 4, 2008, pp. 457-472.

[2] A.G. Polak, D. Wysoczański, J. Mroczka: "A multi-method approach to measurement of respiratory system mechanics”. Metrol. Meas. Syst., vol. XIII, no. 1, 2006, pp. 3-17.

[3] I. Jabłoński, J. Mroczka: "Computer-aided evaluation of a new interrupter algorithm in respiratory mechanics measurement”. Biocybernetics and Biomedical Engineering, 2006 vol. 26, no. 3, pp. 33-47.

[4] I. Jabłoński J. Mroczka: "Frequency-domain identification of the respiratory system model during the interrupter experiment". Measurement, vol. 42, no. 3, 2009, pp. 390-398.

[5] I. Jabłoński, J. Mroczka: "Frequency indexes of respiration during interrupter experiment". Metrol. Meas. Syst., vol. XV, no. 2, 2008, pp. 153-163.

[6] D.W. Kaczka, E.P. Ingenito, K.R. Lutchen: "Technique to Determine Inspiratory Impedance during Mechanical Ventilation: Implications for Flow Limited Patients". Annals of Biomedical Engineering, no. 27, 1999, pp. 340-355.

[7] P.F. Baconnier, P. Carry, A. Eberhard, J. Perdrix, J. Fargnoli: “A computer program for automatic measurement of respiratory mechanics in artificially ventilated patients". Computer Methods and Programs in Biomedicine, vol. 47, 1995, pp. 205-220.

[8] A.G. Polak, J. Mroczka: "Nonlinear model for mechanical ventilation of human lungs". Computers in Biology and Medicine, vol. 36, 2006, pp. 41-58.

[9] J. Gajda, P. Piwowar: "Identification of the human respiratory system during experiment with negative pressure impulse excitation”. Metrol. Meas. Syst., vol. XVI, no. 4, 2009, pp. 569-582.

[10] J.H.T. Bates, P. Baconnier, J. Milic-Emili: "A theoretical analysis of interrupter technique for measuring respiratory mechanics”. J. Appl. Physiol., vol. 64, 1988, pp. 2204-2214. 
[11]K.R. Lutchen, K. Yang, D.W. Kaczka, B. Suki: "Optimal ventilation waveforms for estimating lowfrequency respiratory impedance”. J. Appl. Physiol., vol. 75, 1993, pp. 478-488.

[12] A.B. Dubois, A.W. Brody, D.H. Lewis, B.F. Burgess Jr.: "Oscillation mechanics of lungs and chest in man". J. Appl. Physiol., vol. 8, 1956, pp. 587-594.

[13] K.R. Lutchen, B. Suki: "Understanding pulmonary mechanics using the forced oscillations technique". Bioengineering Approaches to Pulmonary Physiology and Medicine, ed. Khoo, Plenum Press, New York 1996, pp. 227-253.

[14] J. Von Neergaard, K. Wirz: „Die Messung der Strömungswiderstände in den Atemwegen des Menschen, insbesondere bei Asthma und Emphysem“. Z. Klin. Med., no. 105, 1927, pp. 51-82.

[15]G. Liistro, D. Stanescu, D. Rodenstein, C. Veriter: "Reassessment of the interruption technique for measuring flow resistance in humans". J. Appl. Physiol., no. 67, 1989, pp. 933-937.

[16] I. Jabłoński, J. Mroczka: “A forward model of the respiratory system during airflow interruption”. Metrol. Meas. Syst., vol. XVI, no. 2, 2009, pp. 219-232.

[17] J. Mroczka, D. Szczuczyński: "Inverse problems formulated in terms of first-kind Fredholm integral equations in indirect measurements”. Metrol. Meas. Syst., vol. XVI, no. 3, 2009, pp. 333-357.

[18] I. Jabłoński, J. Mroczka: "A station for the respiratory mechanics measurement by occlusion techniques". Metrol. Meas. Syst., vol. XIV, no. 2, 2007, pp. 229-240.

[19]K. Jakuszkin, J. Mroczka: „Analiza właściwości modelu mechanicznego płuc wykorzystująca technikę zoptymalizowanej fali wentylacyjnej”. Modelowanie i Pomiary w Medycynie, 2009, Krynica. (in Polish) 